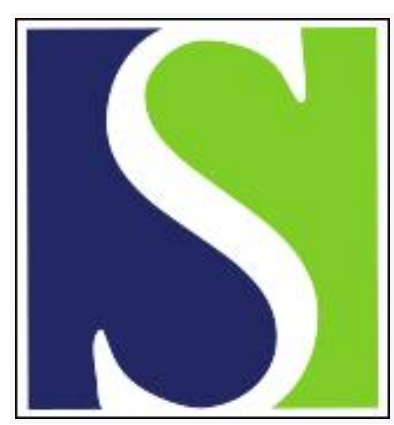

Scand J Work Environ Health 1987;13(4):309-312

https://doi.org/10.5271/sjweh.2047

Issue date: Aug 1987

Centrally and locally mediated vasomotor activities in Raynaud's phenomenon.

by Olsen $\mathrm{N}$

Affiliation: Department of Clinical Physiology and Nuclear Medicine, Rigshospitalet, Copenhagen, Denmark.

This article in PubMed: www.ncbi.nlm.nih.gov/pubmed/3324311

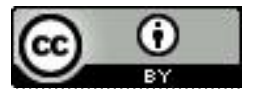




\title{
Centrally and locally mediated vasomotor activities in Raynaud's phenomenon
}

\author{
by Niels OIsen, $M^{1}$
}

\begin{abstract}
OLSEN N. Centrally and locally mediated vasomotor activities in Raynaud's phenomenon. Scand J Work Environ Health 13 (1987) 309-312. A selective review on the attack of Raynaud's phenomenon in primary Raynaud's phenomenon and vibration-induced white finger is presented with special reference to the location of the primary vasospasm, the nature of the dominant pathophysiological mechanism, and the anatomic location of the abnormal link in this mechanism. It is suggested that the primary vasospasm is a total closure of the main digital arteries, that the arterial spasm is mainly mediated through an exaggerated central sympathetic reflex mechanism, and that the abnormal link in this reflex arch could be the smooth muscle cells of the digital arteries. Other anatomic locations of the abnormal link can however not be excluded as yet.
\end{abstract}

Key terms: pathophysiology, primary Raynaud's phenomenon, vibration-induced white finger.

Since Maurice Raynaud (13), in his thesis of 1862, described the disease now named after him, conflicting theories have been advanced concerning the pathophysiology of the characteristic attacks of the disease. In the present paper a selective review of the topic is given with reference to the attacks in vibration-induced white finger (VWF) and in primary Raynaud's phenomenon.

\section{Visible symptoms of the attack}

Raynaud (13) described an exaggerated response of digital circulation to cold exposure or mental stress. The response, denoted as Raynaud's phenomenon, is characterized by the appearance of an acral, welldemarcated blanching or cyanosis in the skin of the finger. It has often been the rule for writers to state that the sequence of colors displayed in the attack is first white, then blue, and finally red in the stage of recovery. However Thomas Lewis (4), in 1929, made detailed observations on the phenomenon and noticed that the attack always started with the cyanotic color in the cooled finger when the finger was held at rest. Microscopically he observed a cessation of the skin capillary blood flow at this stage. The color could change from cyanosis to white spontaneously or as a result of flexing and extending the finger. Full cyanosis in a cooled finger is thus the cardinal sign of the attack, whereas blanching occurs by passive or active mechanisms secondary to the basic mechanism that produces the cyanotic color. The observations by Lewis (4) indicate that the visible symptoms of Raynaud's

\footnotetext{
I Department of Clinical Physiology and Nuclear Medicine, Rigshospitalet, Copenhagen, Denmark.
}

Reprint requests to: Dr N Olsen, Department of Clinical Physiology and Nuclear Medicine, Rigshospitalet, DK-2100 Copenhagen, Denmark. phenomenon reflect an underlying closure of the larger vessels, followed by a closure of the smaller cutaneous vessels.

\section{Arteriovenous anastomoses}

Lewis (5) also discovered the existence of the so-called hunting reaction in normal subjects. The hunting reaction is a vasodilatation that occurs in the skin of the finger after the latter has been immersed in cold water for some time. The reaction is believed to be a local protective mechanism against overcooling and reflects periods with totally opened arteriovenous anastomoses, counteracting the normal sympathetic vasoconstrictor response to cold. Lewis (4) also observed that the hunting reaction could always be found in subjects with Raynaud's phenomenon, although the reaction is far less marked than in normal subjects due to the counteracting spasm of the vessels. An attack of Raynaud's phenomenon therefore is not caused by a lack of the hunting reaction mechanism. The statement that arteriovenous anastomoses run parallel to the capillaries and not in series with them also shows that closure of the arteriovenous anastomoses cannot explain the cessation of capillary blood flow in an attack of Raynaud's phenomenon.

\section{Arterial and venous vasoconstriction theories}

Lewis (4) felt that the primary vasospasm was located on the arterial side of the capillaries and that the digital arteries were the vessels primarily involved. However a venous constriction theory also existed and still exists. The microvascular pressure measurements of Landis (3) in 1930 casts however considerable doubt on the venous constriction theory and seems to be entirely compatible with the arterial constriction theory. Landis (3) measured capillary blood pressure during an attack of 
Raynaud's phenomenon. The capillary blood flow ceased during the attack, and an abnormally low capillary blood pressure was measured. Recovery was registered as the resumption of capillary blood flow, accompanied by an enormous rise in capillary blood pressure and a sudden change in skin color from cyanosis to bright redness, followed by the return of pulse pressure and an increase in skin temperature. If venoconstriction had been primarily responsible for flow cessation, the capillary blood pressure should have been higher and not lower than the habitual pressure. Closure of the vessels located on the venous side of the capillary network may occur, but is then a closure secondary to the basic closure on the arterial side.

\section{Main digital arteries}

From his observations Lewis (4) concluded that the attack was caused by a complete closure of the main digital arteries but was unable to measure it. In 1977 Nielsen \& Lassen (8) described a method to measure the vasoconstrictor response of the digital arteries to cold. The systolic blood pressure of the finger is recorded by this method indirectly with a cuff and strain gauge technique after local cooling of the intermediate phalanx of the finger. The decrease in blood pressure recorded after the cooling is a measure of the increase in the digital arterial tone. An attack of Raynaud's phenomenon is reflected as an unmeasurerable recording, a so-called zero pressure, indicating complete closure of all main digital arteries. The method has an acceptable level of reproducibility, sensitivity, and specificity. Olsen \& Nielsen (11) first applied a modified form of the method, with finger cooling to 30,15 , and $6^{\circ} \mathrm{C}$ combined with the body cooling that is generally necessary to provoke an attack of Raynaud's phenomenon (2), to subjects exposed to hand-arm vibration. Among forestry workers they (12) found that vibration-exposed subjects at stage 0 (Taylor's index) had a decreased response to cold in comparison to those at stages 2 and 3 but an increased response in comparison with that of referents. The results indicated that vibration induces a hyperresponsiveness to cold even before subjects notice any attack of Raynaud's phenomenon. Therefore, after stepwise exclusion of the smaller cutaneous vessels, the arteriovenous anastomoses, and the vessels on the venous side of the capillary network as the location of the primary closure in an attack of Raynaud's phenomenon, strong indications remain that the primary closure is a complete closure of the main digital arteries and not a closure of the smaller arteries, arterioles, or precapillary sphincters.

\section{Anatomic changes in the digital arteries}

From bioptic material Lewis (6) concluded that, in cases of intermittent vasospasm, exemplified by the mildest form of the so-called "Raynaud's disease" (today denoted as primary Raynaud's phenomenon), there is no more intimal thickening than is found in the arteries of warm-handed people of similar age. Hyperplasia of the media was not found in these patients. He found no evidence that attacks of digital arterial spasm lead to thickening of the medial or intimal coats of the digital arteries, but did find that, in advanced cases, attacks may lead to thrombotic events. In advanced cases of VWF the episodies of blanching are replaced by a continuous cyanotic appearance of the fingers, the ability of the blood vessels to constrict and dilate being lost, but only in less than $1 \%$ of patients does this phenomenon lead to malnutrition, tissue necrosis, and gangrene of the fingers (14). Ashe \& Williams (1) studied biopsies from digital arteries in miners with VWF and in dead referents. Subintimal fibrosis occurred in severe cases but was absent in milder cases and in the referents. In the cases without subintimal fibrosis a so-called medial hypertrophy was described that was evaluated from the relationship between wall thickness and lumen diameter. The wall thickness was however equal for the miners and referents, whereas the lumen was largest in the referents, the result being a smaller ratio. They concluded that no observable anatomic changes were seen in the arteries in the mild and moderate cases.

Therefore, it seems that no anatomic changes occur in mild and moderate cases of primary Raynaud's phenomenon and VWF, the attack of Raynaud's phenomenon being a purely vasospastic phenomenon.

\section{Possible mechanisms in the vasospastic attack}

The digital arterial closure in response to cold exposure might be due to (i) central sympathetic reflex mechanisms, (ii) anatomic changes of the arterial wall or lumen, (iii) local nervous mechanisms, (iv) mechanisms of circulating vasoactive substances, (v) mechanisms of locally released vasoactive substances other than those released from nerve endings, (vi) intrinsic myogenic mechanisms, or (vii) intrinsic passive mechanisms.

Centrally mediated vasomotor activities are, in the present paper, synonymous with central sympathetic reflex mechanisms. Locally mediated vasomotor activities are synonymous with the rest of the possible aforementioned mechanisms. Raynaud (13) in 1862 suggested that the vasospasm was mediated through central sympathetic nervous impulses. Contradictory to him Lewis (4) advanced the theory that the attack in Raynaud's phenomenon is produced directly by a local mechanism without the intervention of central sympathetic nervous impulses. Together the antagonistic hypotheses of Raynaud and Lewis constitute the classical theories of the pathophysiological mechanism in the attack of Raynaud's phenomenon. 


\section{Centrally versus locally mediated vasomotor activities}

Lewis performed experiments that provided obvious evidence for his theory. His first direct evidence (4) was the observation that complete anesthetization of the ulnar nerve did not prevent the digital arteries from being subsequently thrown into a complete spasm by local application of cold on the fifth finger. However he only performed this experiment on two of his patients, both severe cases with dry gangrene of the fingers. Lewis (4) felt that, in milder cases of Raynaud's phenomenon, the impossibility of provoking an attack during local anesthesia was due to the lack of a normal central sympathetic vasoconstrictor response, but he could not prove this hypothesis by measurements. Olsen and his co-workers (10) investigated the sympathetic and local vasoconstrictor response to cold in VWF using the Nielsen-Lassen method (8) in its modified form (11). The sympathetic vasoconstrictor response to cold was estimated to be at least twice as large as the locally mediated response. Contradictory to the findings of Lewis (4), and in agreement with those of Raynaud (13), these results indicated that the digital arterial closure is mainly mediated through an exaggerated central sympathetic reflex mechanism. The same results have been obtained by the same method using stellate blockade in subjects with primary Raynaud's phenomenon (9).

Lewis' second direct evidence for a local mechanism in the attack of Raynaud's phenomenon concerned observations on sympathectomized patients (7). He observed a return of the attacks $2-10 \mathrm{~d}$ after the operation in patients with Raynaud's phenomenon with and without nutritional changes of the skin of the fingers. He also observed that the full vasodilatation resulting from preganglionic sympathectomy declines during a period of about one week following an operation. Lewis concluded that sympathectomy does not bring the fingers to a common state; it relieves in all cases, but can be displayed in a measure that is related to the abnormality displayed before the operation. It is however important to notice that none of his cases showed attacks on the first day after sympathectomy. Nielsen et al (9) studied two cases of primary Raynaud's phenomenon without trophic changes by measurements of systolic blood pressure in the finger during combined local and general cooling. Both patients underwent bilateral upper thoracic sympathectomy. The normal response on day 1 after the operation indicated that the attack is dominated by central sympathetic reflex mechanisms. The subsequent return of attacks may indicate that other mechanisms then dominate.

Though a central sympathetic mechanism seems to be the dominant mechanism of an attack, locally mediated mechanisms are not necessarily without influence. It is reasonable to assume that, in milder cases of Raynaud's phenomenon, the attack is the result of both types. It only means that the central sympathetic reflex mechanism makes the greatest and most pathological contibution to the digital arterial constrictor response to cold during a vasospastic attack.

\section{Abnormal link in the central sympathetic reflex arch}

The "receptor site" of the central sympathetic reflex is probably located in the cutaneous thermoreceptors, as it is necessary to perform a local cooling of the finger to provoke an attack of Raynaud's phenomenon. The "effector site" of the reflex is located in the smooth muscle cell of the cooled digital arteries. This reflex arch exists and functions in both normal subjects and in subjects with severe and milder types of Raynaud's phenomenon. The question is now in which part of the reflex arch including receptors and effectors is the fault located in milder cases of Raynaud's phenomenon? Raynaud (13) suggested that the fault was located in the vasomotor centers of the sympathetic nervous system. Lewis (4), who advanced the theory that the mechanism was a local one, found that the fault was located in the digital arteries, probably in the muscular part of the arterial wall. This location is however also exactly where the "effector site" of the central sympathetic reflex arch is placed. However, as seen from newer models of vasoconstriction (15), the fault may be located in many other anatomic areas of the reflex arch, eg, the thermoreceptors, the afferent nerve fibers, the central vasomotor centers, the efferent sympathetic nerve fibers, and the neuromuscular synapses (including pre- and postsynaptic receptors or inside the smooth muscle cell). A functional defect in any of the links of the reflex arch may theoretically produce an abnormal contraction of the digital arteries.

The evidence for a central sympathetic reflex mechanism $(9,10)$ also implies that the initial basic fault in primary Raynaud's phenomenon and VWF is not caused by local axon reflexes, circulating or locally released vasoactive substances, immunologic reactions, viscosity of the blood, systemic blood pressure, or anatomic changes in the intima and lumen of the digital arteries.

\section{Conclusions}

The present selective review on primary Raynaud's phenomenon and VWF leads to the following conclusions:

1. The primary vasopasm is a total closure of the main digital arteries with complete cessation of blood flow.

2. The arterial closure is mainly mediated through an exaggerated central sympathetic reflex mechanism. 
3. The abnormal link in this reflex arch is possibly the smooth muscle cells of the digital arteries, but other anatomic locations of the abnormal link cannot yet be excluded.

\section{References}

1. Ashe WF, Williams N. Occupational Raynaud's II. Arch Environ Health 9 (1964) 425-433.

2. Hellstrøm B, Myhre K. A comparison of some methods of diagnosing Raynaud phenomena of occupational origin. Br J Ind Med 28 (1971) 272-279.

3. Landis EM. Micro-injection studies of capillary blood pressure in Raynaud's disease. Heart 15 (1930) 247-255.

4. Lewis T. Experiments relating to the peripheral mechanism involved in spasmodic arrest of the circulation in the fingers, a variety of Raynaud's disease. Heart 15 (1929) 7-101.

5. Lewis T. Observations upon the reactions of the vessels of the human skin to cold. Heart 15 (1930) 177-208.

6. Lewis $T$. The pathological changes in the arteries supplying the fingers in warm-handed people and in cases of so-called Raynaud's disease. Clin Sci 3 (1938) $287-319$.

7. Lewis T. Raynaud's disease and preganglionic sympathectomy. Clin Sci 3 (1938) 321-336.
8. Nielsen SL, Lassen NA. Measurement of digital blood pressure after local cooling. J Appl Physiol 43 (1977) 907-910.

9. Nielsen SL, Olsen N, Henriksen O. Cold hypersensitivity after sympathectomy for Raynaud's disease. Scand J Thorac Cardiovasc Surg 14 (1980) 109-111.

10. Olsen N, Fjeldborg P, Brøchner-Mortensen J. Sympathetic and local vasoconstrictor response to cold in vibration induced white finger. $\mathrm{Br} \mathrm{J}$ Ind Med 42 (1985) $272-275$.

11. Olsen N, Nielsen SL. Diagnosis of Raynaud's phenomenon in quarrymen's traumatic vasospastic disease. Scand J Work Environ Health 5 (1979) 249-256.

12. Olsen N, Nielsen SL, Voss P. Cold response of digital arteries in chain saw operators. Br J Ind Med 39 (1982) $82-88$.

13. Raynaud M. De l'asphyxie locale et de la gangrene symetrique des extrémités. Rignoux, Paris 1862.

14. Taylor W, Brammer AJ. Vibration effects on the hand and arm in industry: An introduction and review. In: Brammer AJ, Taylor W, ed. Vibration effects on the hand and arm in industry. John Wiley \& Sons, New York, NY 1982, pp 1-12.

15. Vanhoutte PM. Physical factors of regulation. In: Bohr DF, Somlyo AP, Sparks HV, ed. Handbook of physiology. Section 2: The cardiovascular system, volume II. American Physiological Society, Bethesda, MD 1980, pp $443-474$. 